\title{
La dexametasona redujo la mortalidad de pacientes COVID-19 en ventilación mecánica invasiva u oxigenoterapia
}

\author{
Dexamethasone reduced the mortality of COVID-19 patients on invasive mechanical ventilation or oxygen therapy
}

\section{Comentado de:}

Horby P, et al. medRxiv 2020. doi:10.1101/2020.06.22.20137273 ${ }^{1}$

\section{Objetivo}

El objetivo primario del estudio RECOVERY es evaluar la eficacia de los corticosteroides entre otros múltiples tratamientos sobre la mortalidad por todas las causas en pacientes con COVID-19 a los 28 días de seguimiento ${ }^{2,3}$.

\section{Diseño y lugar}

Ensayo controlado aleatorizado abierto, factorial, de cinco terapias para pacientes hospitalizados con COVID-19 en 176 hospitales del Servicio Nacional de Salud (NHS, por sus iniciales en inglés) en el Reino Unido y coordinado por el Departamento de Salud Poblacional de la Universidad de Oxford (ensayo RECOVERY).

\section{Pacientes}

Los pacientes eran elegibles si tenían sospecha clínica o confirmación por laboratorio de infección por SARS-CoV-2, sin antecedentes médicos que implicaran riesgo significativo adicional.

\section{Intervención}

Se realizó una aleatorización centralizada mediante un sistema web que enmascaraba la asignación, en una relación 1:2 a dexametasona $6 \mathrm{mg}$ diarios (por vía oral o intravenosa) durante un máximo de 10 días (o hasta el alta si esta ocurría antes) o bien a cuidados usuales, administrados por los médicos tratantes (las otras ramas activas fueron lopinavir-ritonavir, azitromicina, plasma de convaleciente o tocilizumab, pero no todas las opciones estaban disponibles todo el tiempo).

\section{Medición de resultados principales}

El resultado primario fue mortalidad a los 28 días. Los resultados secundarios incluyeron el tiempo al alta hospitalaria y, entre quienes no estaban en ventilación mecánica invasiva (VMI) al inicio, el resultado combinado de muerte o necesidad de VMI o de membrana de oxigenación extracorpórea (ECMO, por sus iniciales en inglés). Para el resultado primario y la estadía hospitalaria se utilizó Hazard Ratio de Cox y las curvas de sobrevida de
Kaplan-Meier, y para el resultado compuesto, un modelo de regresión binomial. Los análisis preespecificados de subgrupos fueron: edad, sexo, nivel de soporte respiratorio, días de síntomas y riesgo basal de mortalidad.

El tamaño muestral no había sido calculado al comienzo del estudio, pero en su transcurso se determinó que, si la mortalidad a 28 días fuera de $20 \%$, incluyendo al menos 2.000 pacientes a una rama activa y 4.000 a cuidados usuales, tendría al menos $90 \%$ de poder ( $\mathrm{P}=0,01$ a dos colas) para detectar una diferencia absoluta, clínicamente relevante, de $4 \%$ entre los grupos. En consecuencia, tras superar los 2.000 pacientes, el 8 de junio de 2020 el Comité Directivo cerró el reclutamiento del brazo dexametasona.

\section{Resultados principales}

En esta comparación se incluyeron 6.425 pacientes. La edad promedio fue de 66,1 años, $36 \%$ eran mujeres y $56 \%$ presentaban al menos una comorbilidad importante. La aleatorización balanceó apropiadamente los grupos, aunque la edad promedio fue 1,1 años mayor en el grupo dexametasona. Se compararon 2.104 pacientes asignados a dexametasona (95\% recibió al menos una dosis) con 4.321 pacientes asignados a la cuidados usuales (7\% recibió dexametasona). Se documentó la muerte dentro de los 28 días de 454 (21,6\%) pacientes del grupo dexametasona y 1065 (24,6\%) del grupo cuidados usuales (Riesgo Relativo [RR] ajustado por edad 0,83; Intervalo de confianza [IC] del $95 \% 0,74$ a 0,$92 ; p<0,001)$. Los pacientes sintomáticos por más de 7 días (que eran más propensos a VMI) tuvieron un mayor beneficio en este desenlace.

Al momento de la aleatorización, $16 \%$ de los participantes recibía VMI o ECMO, $60 \%$ recibía sólo oxígeno (con o sin ventilación mecánica no invasiva), mientras que $24 \%$ no estaban recibiendo soporte respiratorio. El efecto varió significativamente dependiendo del nivel de soporte respiratorio basal (ver Figura 1).

Fuente de financiamiento: Este estudio fue financiado por el Consejo de Investigación Médica e Instituto Nacional de Investigación en Salud del Reino Unido (Subvención MC PC 19056).

Figura 1. Efecto de la dexametasona en la mortalidad a 28 días, según el soporte respiratorio basal de los participantes. Fuente: traducido de Horby P, et al. medRxiv 2020:2020.06.22.20137273. Nota: IC: intervalo de confianza; RR: riesgo relativo; VMI: ventilación mecánica invasiva

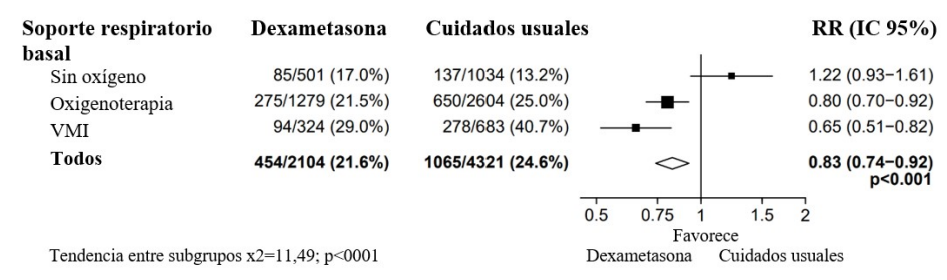




\section{Comentario}

Estos resultados preliminares muestran que la dexametasona reduce la mortalidad en los pacientes con COVID-19 que reciben VMI en aproximadamente un tercio, y en un quinto en pacientes que reciben oxigenoterapia sin VMI. La tasa de mortalidad del grupo control fue consistente con la letalidad hospitalaria en el Reino Unido, sugiriendo la representatividad de los resultados ${ }^{4}$. El riesgo de sesgo evaluado con la herramienta CochraneRoB2 fue bajo para el desenlace de mortalidad, si bien podría haber algunas preocupaciones para los resultados secundarios, pues por tratarse de un estudio abierto (es decir, no ciego) existiría la posibilidad de desviaciones del protocolo. Sin embargo, estas desviaciones no ocurrieron, o en todo caso, hubieran reducido las diferencias, debido a que $7 \%$ del grupo control recibió dexametasona. El beneficio fue más claro en los pacientes tratados luego de más de siete días desde el inicio de los síntomas, cuando el daño pulmonar inflamatorio es mayor. Sin embargo, no hubo beneficio, e incluso se observaron posibles daños, en pacientes hospitalizados que no necesitablan soporte respiratorio.

Este gran ensayo pragmático, que incluyó alrededor de $15 \%$ de todos los pacientes hospitalizados en el Reino Unido por esta enfermedad, permitió una evaluación rápida y sólida del impacto de los posibles tratamientos disponibles para COVID-19 sobre la mortalidad a 28 días. Sólo se recopilaron datos esenciales, y la información adicional (incluida la mortalidad a largo plazo) provino de fuentes rutinarias. Las experiencias exitosas, como ésta, debieran ser replicadas en el resto del mundo para COVID-19 y otras patologías.

La respuesta inmune del huésped desempeña un papel clave en la fisiopatología de la insuficiencia orgánica en las neumonías virales graves ${ }^{5-7}$. La COVID-19 grave no es la excepción, tal como lo sugieren los marcadores inflamatorios notablemente elevados como la proteína $C$ reactiva, la ferritina y las interleuquinas 1 y $6^{8-11}$. Además, el mayor beneficio en mortalidad se observó en una etapa tardía de la enfermedad, cuando la inmunopatología juega un papel más importante que la replicación activa del virus.

Los corticoides ya se habían utilizado ampliamente en síndromes estrechamente relacionados con COVID-19, incluyendo SARS, MERS, influenza severa y neumonía adquirida en la comunidad, aunque en base a evidencia insuficiente ${ }^{12-15}$. Es posible que la desregulación del sistema renina-angiotensina inducida por la dexametasona en el contexto de SARS-CoV-2 ${ }^{16}$ explique en parte los impactantes resultados, que difícilmente sean extrapolables a otras virosis.

Según los resultados de este estudio, se evitaría una muerte tras el tratamiento de ocho pacientes con VMI, o de 25 pacientes que requieren oxígeno.

Si bien la evidencia resumida proviene de una investigación cuyo reporte todavía no fue evaluado por pares, dado lo alentador de sus resultados, fue considerada para ser incorporada a las guías de manejo de pacientes con enfermedad por SARSCov-2 del Reino Unido, a pesar de que el propio repositorio de preimpresiones desaconseja trasladar tan rápidamente esta información preliminar a recomendaciones clínicas ${ }^{17}$.

\section{Conclusiones del comentador}

Debido al impactante beneficio observado en este estudio, su perfil de seguridad, su bajo costo y su vasta disponibilidad, es posible que la dexametasona sea pronto utilizada ampliamente en todo el mundo para salvar las vidas de los pacientes con COVID-19 más gravemente enfermos, aliviando el estrés de los sistemas de salud, particularmente en los sectores con mayor demanda de cuidados críticos.

Agustín Ciapponi [ Servicio de Medicina Familiar y Comunitaria, Hospital Italiano de Buenos Aires; Cochrane Argentina, Instituto de Efectividad Clínica y Sanitaria. aciapponi@iecs.org.ar ]

Ciapponi A La dexametasona redujo la mortalidad de pacientes COVID-19 en ventilación mecánica invasiva u oxigenoterapia. Evid Actual Pract Ambul. 2020;23(3):e002078. Comentado de: Horby P, et al. Effect of Dexamethasone in Hospitalized Patients with COVID-19 - Preliminary Report. medRxiv 2020. doi:10.1101/2020.06.22.20137273

\section{Referencias}

1. Horby P, Lim WS, Emberson J, et al.. Effect of Dexamethasone in Hospitalized Patients with COVID-19: Preliminary Report. ; 2020. Available from: https://doi.org/10.1101/2020.06.22.20137273 [Last access: 2020-07-10]

2. Randomised evaluation of COVID-19 therapy (RECOVERY). Study protocol [V7.0 2020-06-18]; 2020. Available from: https://www.recoverytrial.net/ files/recovery-protocol-v7-0-2020-06-18.pdf [Last access: 2020-07-10].

3. Randomised Evaluation of COVID-19 Therapy (RECOVERY); 2020. Available from: https://clinicaltrials.gov/ct2/show/NCT04381936 [Last access: 2020-07-10].

4. Docherty AB, Harrison EM, Green CA, et al. Features of 20133 UK patients in hospital with covid-19 using the ISARIC WHO Clinical Characterisation Protocol: prospective observational cohort study. BMJ. 2020;369:m1985. Available from: 10.1136/bmj.m1985.

5. Cameron MJ, Ran L, Xu L, et al. Interferon-mediated immunopathological events are associated with atypical innate and adaptive immune responses in patients with severe acute respiratory syndrome. J Virol. 2007;81(16):8692-8706. Available from: 10.1128/JVI.00527-07.

6. Wong CK, Lam CW, Wu AK, et al. Plasma inflammatory cytokines and chemokines in severe acute respiratory syndrome. Clin Exp Immunol. 2004;136(1):95-103. Available from: 10.1111/j.1365-2249.2004.02415.x.

7. Baillie JK, Digard P. Influenza - Time to Target the Host? N Engl J Med. 2013;369(2):191-193. Available from: 10.1056/nejmcibr1304414

8. Ruan Q, Yang K, Wang W, et al. Correction to: Clinical predictors of mortality due to COVID-19 based on an analysis of data of 150 patients from Wuhan, China. Intensive Care Med. 2020;46(6):1294-1297. Available from: 10.1007/s00134-020-06028-z.

9. Ruan Q, Yang K, Wang W, et al. Clinical predictors of mortality due to COVID-19 based on an analysis of data of 150 patients from Wuhan, China. Intensive Care Med. 2020:46(5):846-848. Available from: 10.1007/s00134-020-05991-x. 
10. Huang C, Wang Y, Li X, et al. Clinical features of patients infected with 2019 novel coronavirus in Wuhan, China. Lancet. 2020;395(10223):497-506 Available from: 10.1016/S0140-6736(20)30183-5.

11. Moore JB, June CH. Cytokine release syndrome in severe COVID-19. Science. 2020;368(6490):473-474. Available from: 10.1126/science.abb8925.

12. Stockman LJ, Bellamy R, Garner P. SARS: Systematic Review of Treatment Effects. PLoS Medicine. 2006;3(9):e343-e343. Available from: 10.1371/ journal.pmed.0030343.

13. Arabi YM, Mandourah Y, Al-Hameed F, et al. Corticosteroid Therapy for Critically III Patients with Middle East Respiratory Syndrome. Am J Respir Crit Care Med. 2018;197(6):757-767. Available from: 10.1164/rccm.201706-1172OC.

14. Lansbury LE, Rodrigo C, Leonardi-Bee J, et al. Corticosteroids as Adjunctive Therapy in the Treatment of Influenza: An Updated Cochrane Systematic Review and Meta-analysis. Critical Care Med. 2020;48(2):e98-e106. Available from: 10.1097/CCM.00000000000004093.

15. Siemieniuk RAC, Meade MO, Alonso-Coello P, et al. Corticosteroid Therapy for Patients Hospitalized With Community-Acquired Pneumonia: A Systematic Review and Meta-analysis. Ann Intern Med. 2015;163(7):519-519. Available from: 10.7326/M15-0715.

16. Liaudet L, Szabo C. Blocking mineralocorticoid receptor with spironolactone may have a wide range of therapeutic actions in severe COVID-19 disease. Crit Care. 2020;24(1):318-318. Available from: 10.1186/s13054-020-03055-6.

17. Whitty C. Dexamethasone in the treatment of COVID-19: Implementation and management of supply for treatment in hospitals; 2020. Available from https://www.cas.mhra.gov.uk/ViewandAcknowledgment/ViewAlert.aspx?AlertID=103054 [Last access: 2020-07-10] 